\title{
Postoperative Pain and Recurrence after Mesh Fixation with Absorbable versus Non-Absorbable Tacks in Patients undergoing Laparoscopic Transabdominal Inguinal Hernia Repair: A Comparative Study
}

\author{
Dina Hany, ${ }^{1}$ Mohamed Gamal ${ }^{2}$ \\ ${ }^{1}$ Department of General Surgery, Ain Shams University, Egypt \\ 2Department of General Surgery, Helwan University, Egypt
}

Introduction: Inguinal hernia is a common disease. Many researches were done to evaluate the different methods of treatment yet there is no ideal method for treatment till now. Since the use of laparoscopy in hernia repair evolved, it is probably becoming the treatment of choice for hernia.

Objectives: This is a prospective randomized trial that aimed at comparing the outcome of using absorbable versus non-absorbable tacks as mesh fixation devices in patients undergoing laparoscopic transabdominal inguinal hernia repair (TAPP) regarding postoperative groin pain and early recurrence.

Patients and Methods: Thirty patients were enrolled in this study. The patients were divided into two groups: Group A included 15 patients who underwent TAPP with mesh fixation by non absorbable tack (NAT) and group B included 15 patients who underwent TAPP with mesh fixation by absorbable tack (AT). Follow up was done at the first day, second week, sixth month and one year postoperatively.

Results: The 2 groups had similar features regarding demographics, operation time, postoperative hospital stay and morbidity. The 2 fixation methods were found similar for postoperative pain and recurrence with no significant difference.

Disclosure: This article is not sponsored by any company, so the authors have no competing interests as defined by Nature Publishing Group, or other interests that might be perceived to influence the results and/or discussion reported in this article.

Key words: Laparoscopic inguinal hernia repair, mesh fixation, pain, recurrence.

\section{Introduction}

Inguinal hernias are one of the most common complaints that surgeons hear from patients on a daily basis. Whether laparoscopic or open repair is the ideal for inguinal hernias is still controversial. Hernia repair can be done by two methods: Total extraperitoneal (TEP) and transabdominal preperitoneal (TAPP) repairs. ${ }^{1}$

The transabdominal preperitoneal (TAPP) repair involves standard laparoscopy with access into the peritoneal cavity and placement of a large mesh along the anterior abdominal wall, thereby repairing the hernia posterior to the defect. This technique was the first laparoscopic hernia repair to be performed. $^{2}$

In TAPP procedure, the mesh must be fixed after its placement either by tacks (titanium or absorbable), sutures, staples, self-fixing meshes or other glues. There is no consensus on the best method of mesh fixation and the choice of options often depends on surgeons' personal preference. ${ }^{3}$

Tackers are very popular fixation devices for inguinal hernia repairs as they are simple to use and prevent mesh migration, which is the most common cause for recurrence. ${ }^{4,5}$ However, one of the biggest issues with tacks is the risk of causing long-term pain after its use. ${ }^{1}$

Recently, the market/surgeon preference is turning toward absorbable spiral tacks which appear to cause less long-term complications than the titanium tacks and tend to reabsorb within one year. ${ }^{1,2}$

Absorbable fixation devices have been developed to achieve sufficient tensile fixation strength with acceptable postoperative pain compared to conventional non absorbable devices. Yet, their efficiency has not been confirmed by randomized controlled clinical trials. ${ }^{6}$

Guidelines of European Association for Endoscopic Surgery and other interventional techniques (EAES) and the European Hernia Society (EHS) reported that, at present, no adequate clinical studies about the use of absorbable devices are available, and they could not make any recommendation. ${ }^{7}$

\section{Objectives:}

In the present study we are comparing the outcome of absorbable versus non-absorbable tacks as mesh fixation devices in patients undergoing laparoscopic transabdominal inguinal hernia repair (TAPP) in regard 
to postoperative chronic groin pain and recurrence.

\section{Patients and methods}

This study was conducted in Eldemerdash Hospital, Ain Shams University and Badr hospital, Helwan University between June 2015 and June 2018. The study included 30 male patients suffering from bilateral inguinal hernia who were candidate for laparoscopic transabdominal preperitoneal repair (TAPP). Patients were divided into two groups by random selection; the first group (group A) included 15 males who underwent TAPP using nonabsorbable tacks (NAT) while the second group (group B) included 15 males who underwent TAPP using absorbable tacks (AT).

Inclusion criteria included patients aged 20-60 years, patients who had bilateral inguinal hernia with American Society of Anesthesiology Scores (ASA) I and II patients and signed informed consent.

Exclusion criteria included patients with history of previous abdominal operations, recurrent hernia, irreducibility, ASA III and IV, BMI $\geq 35$, patients with COPD, emergency presentation, large scrotal hernia, contraindications to general anesthesia and patients who refused to participate in the study.

Cases that underwent conversion were excluded from the study.

Smokers were advised to stop smoking at least one month before the operation. Any predisposing factor for hernia was treated preoperatively.

\section{Surgical technique:}

Patients were instructed to void before surgery, which rendered bladder catheterization unnecessary.

A single preoperative dose of parenteral 3rd generation cephalosporin was administered on anesthesia induction. General anesthesia was administered routinely.

Patients were put in a supine head down leg up position with both arms tucked. Bilateral compression stockings were applied. The table was tilted opposite the side of the hernia operated upon. The anesthesia monitor was placed as far from the head of the table as possible. A single video monitor was placed at the foot of the bed, directly facing the patient's head, with the surgeon standing by the patient's shoulder on the opposite side of the hernia, the assistant surgeon and the nurse standing on the ipsilateral side. The skin is prepared and draped so as to allow exposure of the entire lower abdomen, the genital region, and the upper thighs when necessary.

A pneumoperitoneum was established using a Veress needle in the left subcostal area. Three trocars were used. A $10-\mathrm{mm}$ trocar was placed at the epigastrium just below the sternum to obtain adequate distance from the hernia orifice. A $30^{\circ}$ endoscope was inserted through this $10 \mathrm{~mm}$ trocar. Other trocars for working instruments; one $5 \mathrm{~mm}$ and one $10-\mathrm{mm}$ were inserted under direct visualization at the level of umbilicus just lateral to the lateral border of rectus muscle.

Diagnostic laparoscopy was first done. Identification of the median and medial umbilical ligaments, bladder, inferior epigastric vessels and hernial defects whether direct or indirect or both was done. Marking of the site of peritoneal incision was done from anterior superior iliac spine to medial umbilical ligament. The incision was sufficiently above the hernia defect to allow dissection of 2 to $3 \mathrm{~cm}$ of normal fascia to provide sufficient mesh overlap after mesh placement.

Peritoneal flap elevation was done beginning at the lateral edge of the medial umbilical ligament and extending in a subfascial plane 8 to $10 \mathrm{~cm}$ laterally till the anterior superior iliac spine. The intra-abdominal pressure in this phase helped to divide the peritoneum from the underlying abdominal wall.

After developing the peritoneal flap in the avascular plane between the peritoneum and the transversalis fascia, dissection was done to reach the pubic symphysis, Cooper's ligament, iliopubic tract, cord structures, inferior epigastric vessels, and hernia spaces followed by dissection of the sac from the cord structures.

The most crucial step in TAPP procedure was the accurate preparation of the external borders of the peritoneal flap. This allowed the mesh to be perfectly positioned on the inguinal area, medially, laterally, and in femoral and obturator areas. We used two separate peritoneal incisions for each side. Adherence to the peritoneum was another cruial step to avoid injury to the femoral branch of the genitofemoral nerve and lateral cutaneous nerve of the thigh in the triangle of pain.

For direct hernia gentle reduction of sac from the preperitoneal fat using gentle traction was done. While indirect sacs were mobilized from the cord structures and then reduced into the peritoneal cavity. A larger hernia sac that was difficult to mobilize from the cord without undue trauma to the vas deferens or vasculature to the testicle was divided just distal to the internal ring, leaving the distal sac in situ within the inguinal canal. After dissection was done, the mesh was placed and fixed using either non-absorbable tacks (Group A) or absorbable tacks (Group B), we used 3-5 tacks to fix the mesh for every side and another 3-5 tacks to close the peritoneum. In our study we used the ProTack $\AA$ by Covidien as nonabsorbable tacks (Fig. 1) and AbsorbaTack $®$ by Covidien as absorbable tacks (Fig. 2).

We avoided any gaps when closing the peritoneum to minimize the likelihood of small bowel herniation and obstruction. 


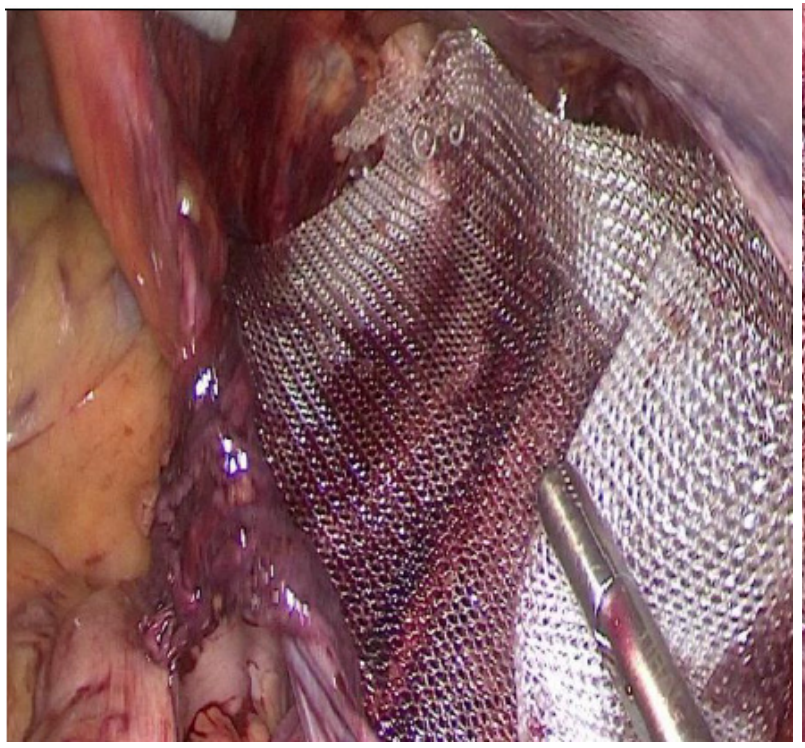

Right side of the hernia

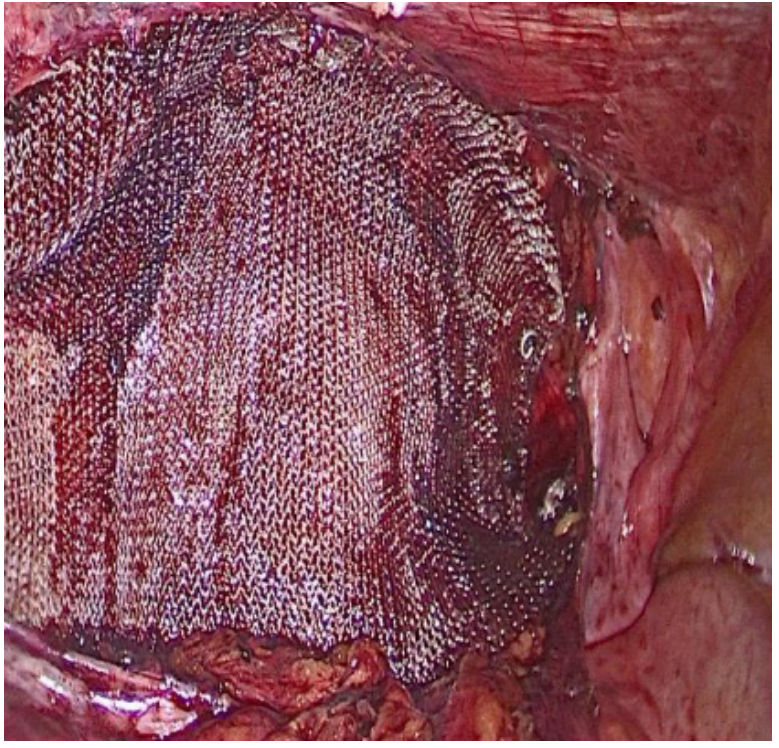

Left side of the herni

Fig 1: Fixing mesh using non-absorbable tacks.

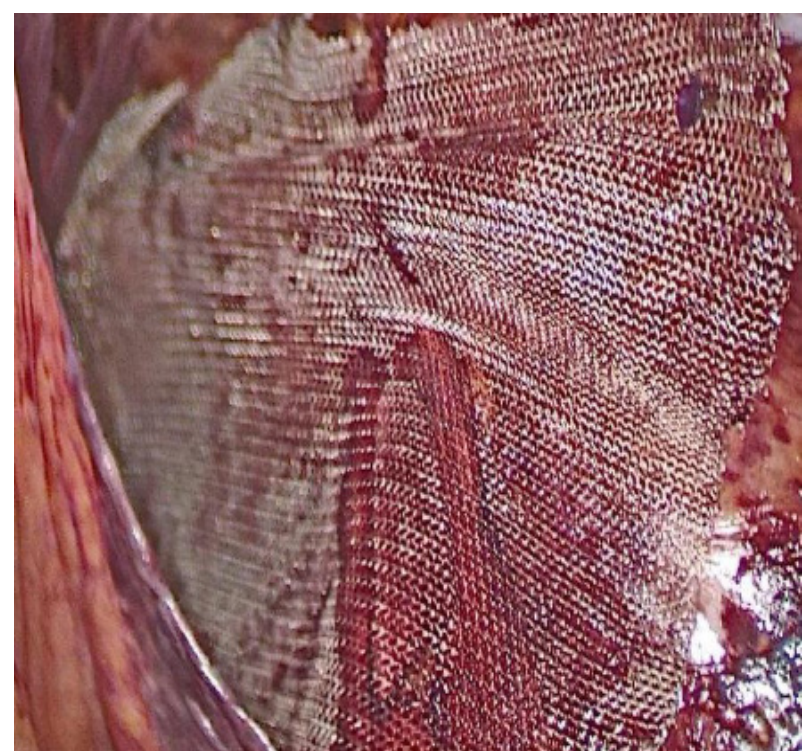

Right side of the hernia

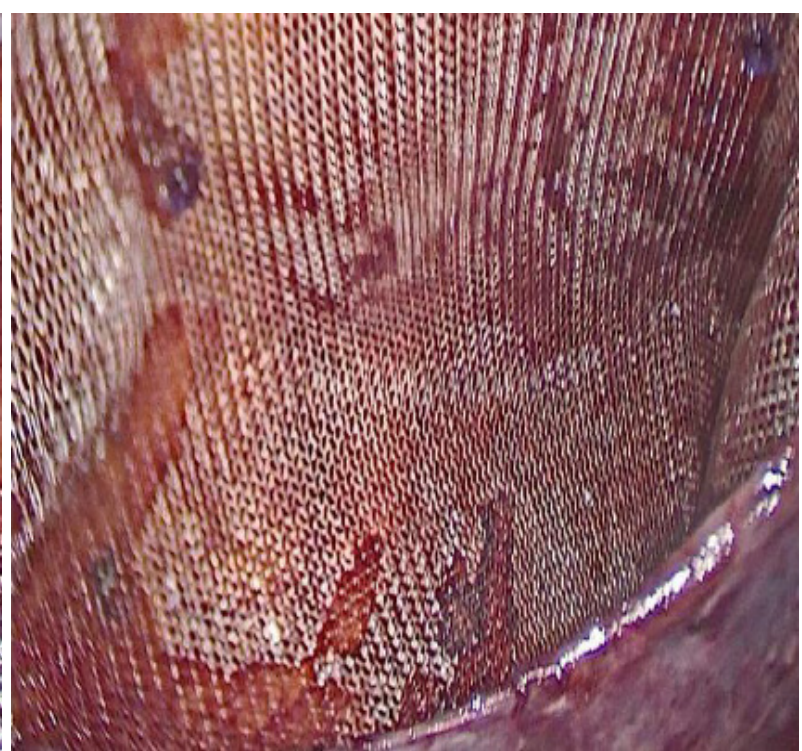

Left side of the hernia

Fig 2: Fixing mesh using absorbable tacks.

Working trocars were removed, deflation of the abdomen was done while the camera was observing the site of repair then the last trocar and camera were removed. Compression at the hernia site at the inguinal region was done. Skin incisions were closed. Ten minutes before the end of procedure, ketolac (30 $\mathrm{mg}$ ) was given intravenously.

All patients received standard postoperative care, including mobilization and return to normal diet as quickly as possible. Analgesia in the form of $1 \mathrm{gram}$ paracetamol was given every 8 hours for 3 days. Reparil tablets ( 2 tablets 3 times daily) were given for 2 weeks.
Discharge was done on the first postoperative day.

\section{Follow-up}

All patients were scheduled to return for an outpatient visit in the second week, sixth month and 1 year after the surgery. The primary outcome measure in this study was pain and recurrence.

Postoperative pain was assessed in first day, second week, sixth month and one year postoperatively both subjectively and objectively. Pain was assessed subjectively by patient's complaint of presence of pain and whether mild, moderate or severe. Assessment of 
pain by visual analogue scale (VAS) score was difficult to apply for all patients in the study. Pain was assessed objectively by Functional Activity Score. This is an activity-related score where pain is assessed when the patient was asked to perform an activity related to their painful area (deep breath and cough and movement of limb on the operated side).

Patients were assessed and score $A, B$, or $C$ was given: $A=$ No limitation; means the patient's activity is unrestricted by pain.

$\mathrm{B}=$ Mild limitation; means the patient's activity is mild to moderately restricted by pain.

$\mathrm{C}=$ Severe limitation; means the patient ability to perform the activity is severely limited by pain.

Any recurrence, chronic groin pain, discomfort and foreign body sensation were recorded. Any seromas or hematomas and scrotal edema were considered postoperative complications when they limited daily activities or required surgical intervention.

\section{Statistical analysis}

Statistical analysis was performed using IBM SPSS Statistics version 20.0 for Windows. Results were compared by Student $t$ test or Mann-Whitney $U$ test for continuous variables, and chi-square or Fisher exact tests were used for categorical variables. A p value $<0.05$ was considered to represent statistical significance.

\section{Results}

Thirty patients (group $A=15$ and group $B=15$ ) were enrolled in the study, all patients were males (100\%).

Demographics and patients' related data:

There was no significant difference in term of age, sex, BMI, ASA score, smoking, weight lifting either heavy exercise or occupational and presenting symptom between the two groups (Table 1).

Table 1: Demographics and patients' related data according to mesh fixation group variables

\begin{tabular}{lccc}
\hline & Group A & Group B & P value \\
\hline Number of patients & 15 & 15 & \\
Age (mean years \pm SD) & $46.33 \pm 9.9$ & $42.13 \pm 10.24$ & 0.263 (NS) \\
Sex M:F & $15: 0$ & $15: 0$ & 0.61 (NS) \\
BMI (mean kg/m2 \pm SD) & $29.4 \pm 3.2$ & $30 \pm 3.16$ & 0.136 (NS) \\
Smoking & 11 & 7 & 0.195 (NS) \\
Weight lifting & 13 & 10 & 0.456 (NS) \\
ASA score & & 5 & 0.456 (NS) \\
I & 7 & 10 & $0.361(\mathrm{NS})$ \\
II & 8 & 4 & 0.624 (NS) \\
Comorbidities & 2 & 3 & $0.361(\mathrm{NS})$ \\
DM & 2 & 3 & \\
HTN & 4 & & \\
Mild restrictive pulmonary function & & 13 & 0.558 (NS) \\
Presenting symptom & 11 & 1 & \\
Swelling & 1 & 1 & \\
Pain & 3 & & \\
both & & & \\
\hline
\end{tabular}

SD: Standard deviation, M: Male, F: Female, BMI: Body Mass Index, ASA: American Society Of Anesthesiologists,

DM: Diabetes mellitus, HTN: Hypertension, NS: non-significant.

\section{Operative data}

Operative data are shown in Table 2. There was no statistical difference between the two groups as regard operative time (Figure 6) and intraoperative complications. The mean operation time was $77.67 \pm 34.33$ minutes in group $A$ and $80.2 \pm 23.88$ minutes in group B. One patient in group A had intraoperative bladder injury during peritoneal dissection which was repaired laparoscopically. The mean number of tacks used was mean $16.87 \pm 3.22$ in group $A$ and $17.4 \pm 2.92$ in group $B$. 


\begin{tabular}{lccc}
\hline & Group A & Group B & P value \\
\hline Operative time (minutes) & $77.67 \pm 34.33$ & $80.2 \pm 23.88$ & 0.816 (NS) \\
Intraoperative complications & 1 & 0 & 0.309 (NS) \\
Number of tacks Mean \pm SD & $16.87 \pm 3.22$ & $17.4 \pm 2.92$ & 0.639 \\
\hline
\end{tabular}

\section{Postoperative data according to mesh fixation group}

Postoperative data are summarized in Table 3. There was no statistically significant difference between both groups in postoperative hospital stay, postoperative complications, readmission, return to normal activity and recurrence (Figure 7). The mean postoperative hospital stay was $1.27 \pm 0.46$ days in group A and $1.2 \pm 0.41$ days in group B. There were no cases of mortality. Postoperative complications included inguinoscrotal edema; 2 patients in group $\mathrm{A}$ and 1 patient in group $B$, all were treated conservatively within 2 weeks. 1 patient in group B had port site infection and this was the only case of readmission for drainage. The mean time for return to normal activity was $5.33 \pm 1.68$ in group A and $5.4 \pm 1.18$ in group B. There was no recurrence in either group.

Table 3: Postoperative data variables

\begin{tabular}{lccc}
\hline & Group A & Group B & P value \\
\hline Hospital stay & $1.27 \pm 0.46$ & $1.2 \pm 0.41$ & 0.679 (NS) \\
Postoperative complications & 2 & 2 & 1 (NS) \\
Inguinoscrotal edema & 2 & 1 & 0.543 (NS) \\
Port site infection & 0 & 1 & 0.309 (NS) \\
Readmission & 0 & 1 & 0.309 (NS) \\
Return to normal activity & $5.33 \pm 1.68$ & $5.4 \pm 1.18$ & 0.901 (NS) \\
Recurrence & 0 & 0 & \\
\hline
\end{tabular}

Postoperative pain assessment according to mesh fixation groups

Postoperative pain disappeared throughout the follow up period with $100 \%$ pain free patients in both groups at 6 months. There was no statistically significant difference between the 2 groups regarding postoperative pain during follow up at the $1^{\text {st }}$ day (Figure 3 ), 2weeks (Figure 4), 6 month (Figure 5) and 1 year (Figure 6) postoperatively.
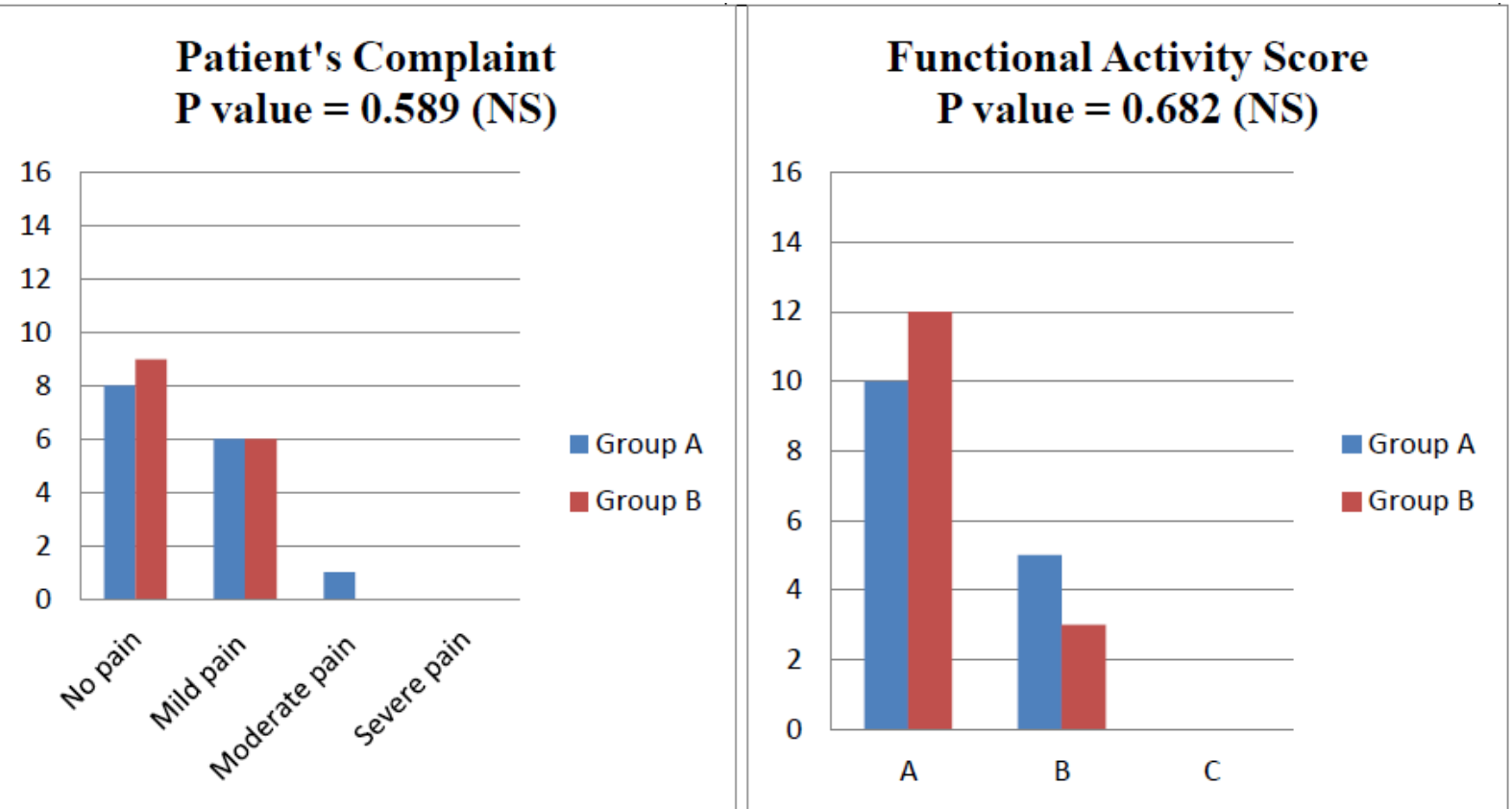

Fig 3: Pain assessment on 1st postoperative day. 


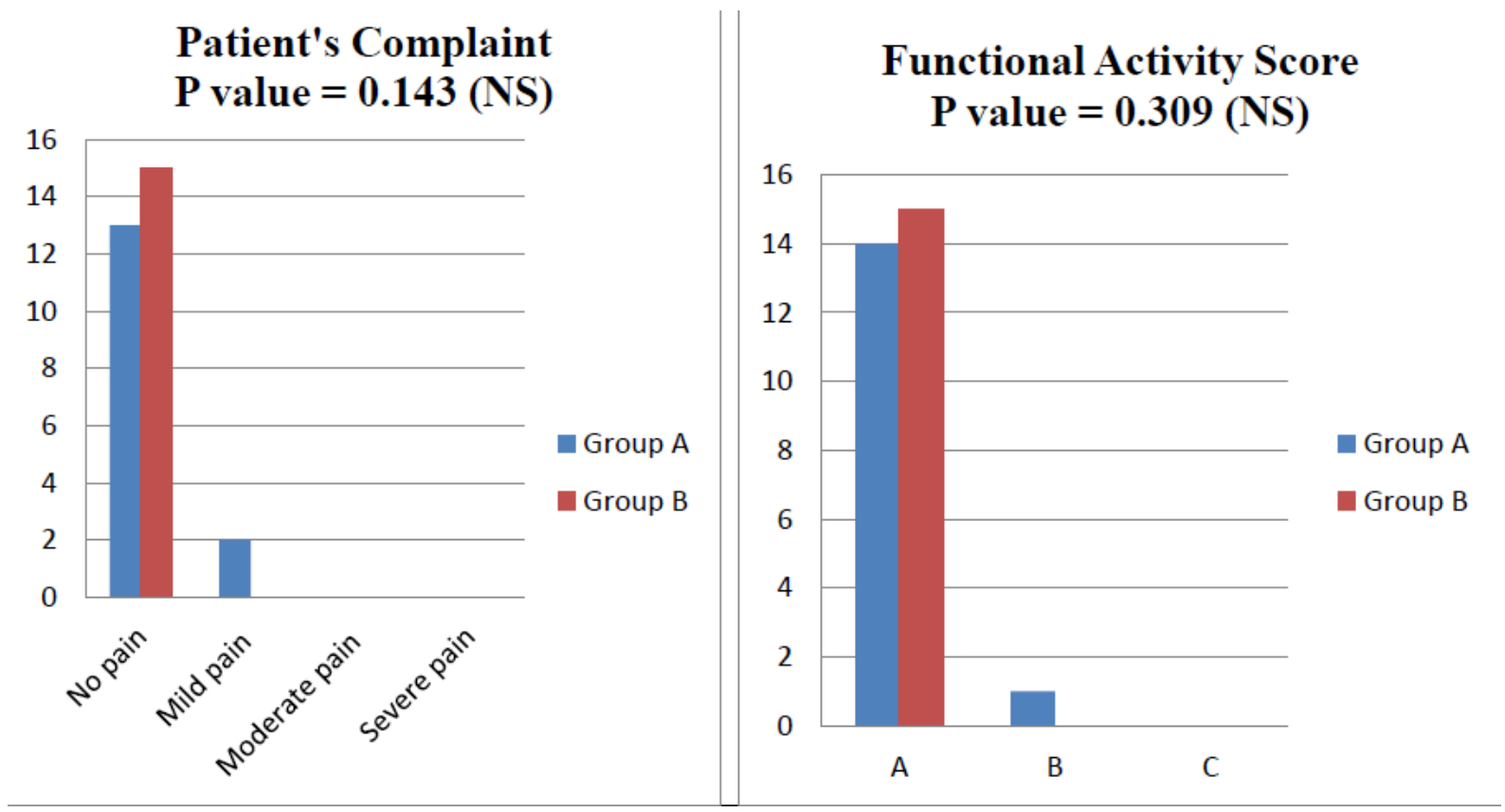

Fig 4: Pain assessment 2 weeks postoperative.

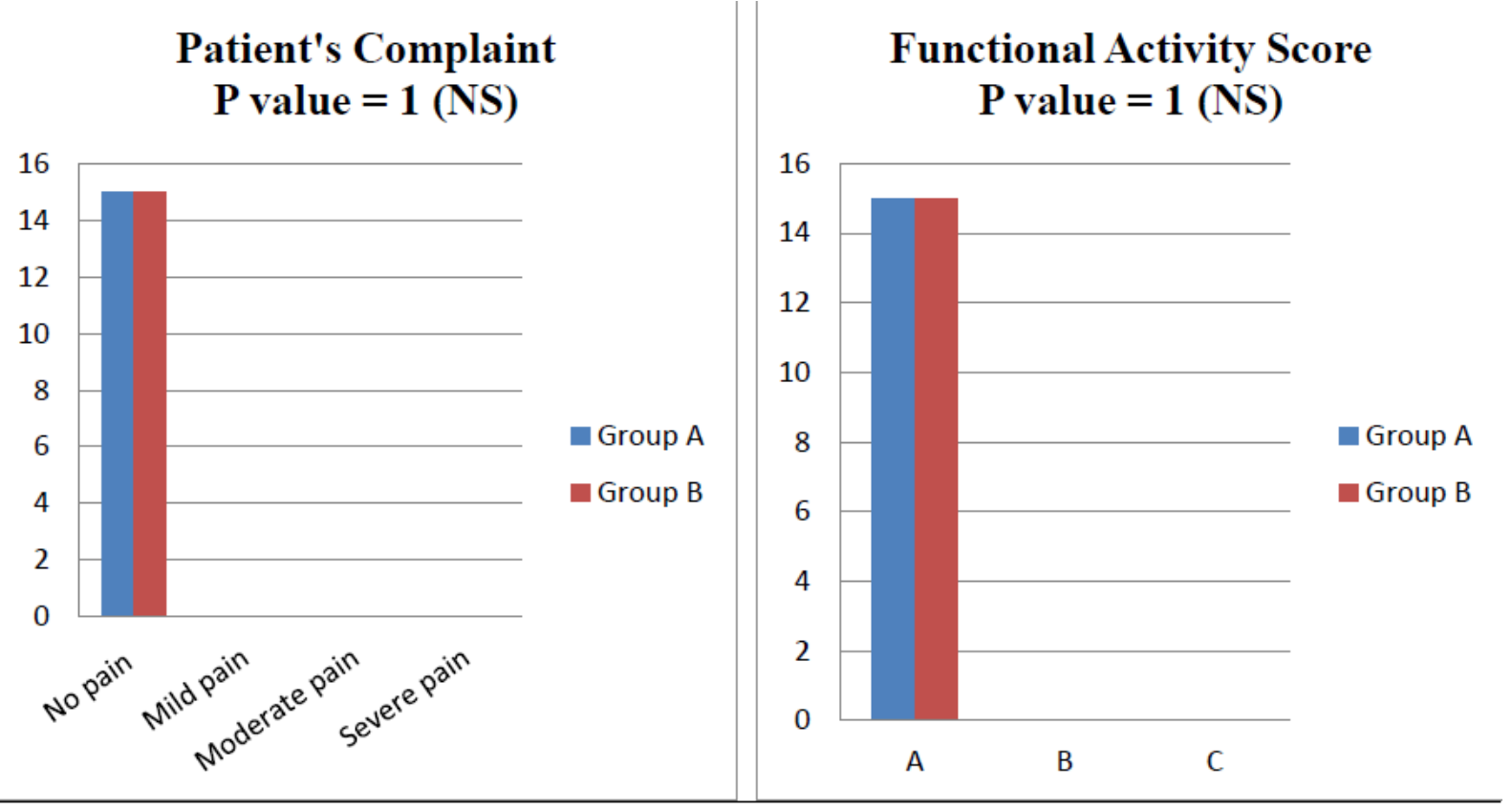

Fig 5: Pain assessment 6 months postoperative. 


\section{Patient's Complaint $P$ value $=1$ (NS)}

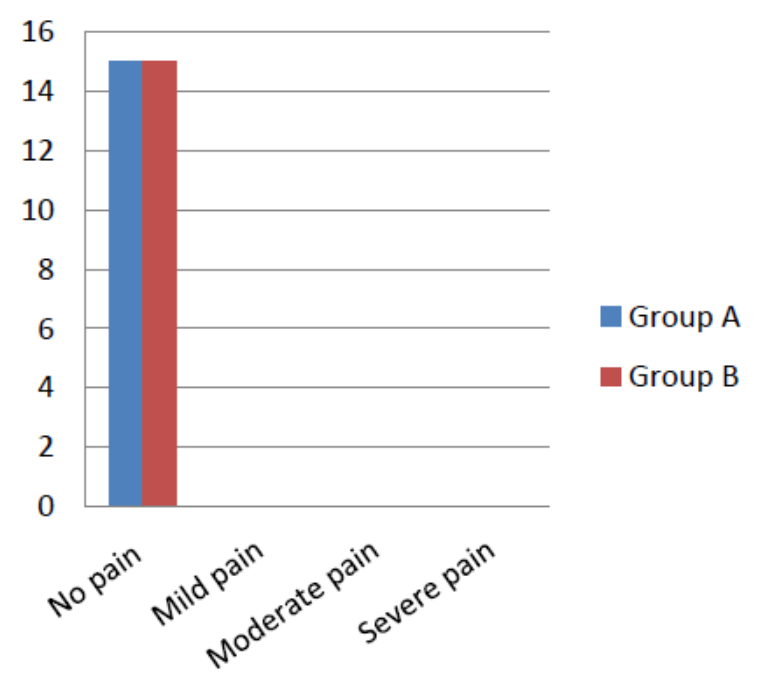

\section{Functional Activity Score $P$ value $=1($ NS)}

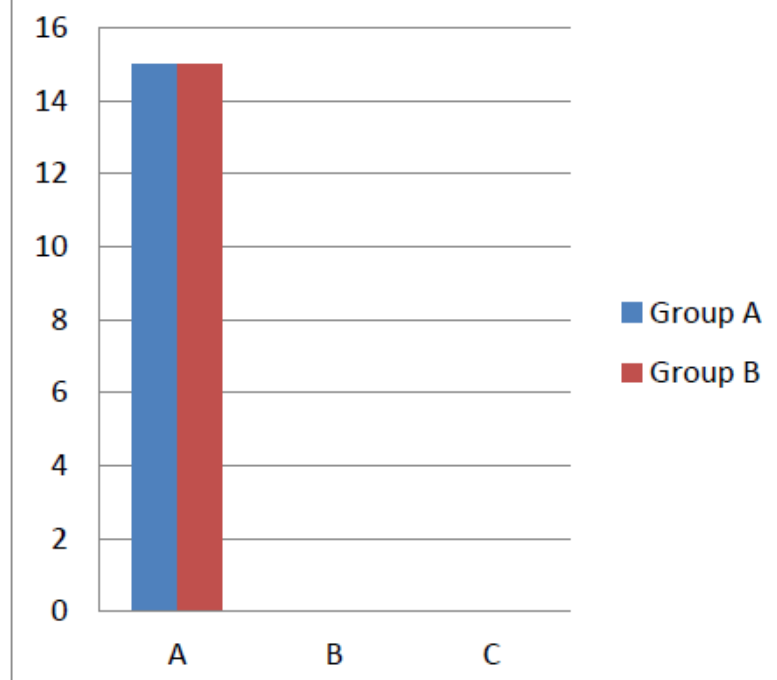

Fig 6: Pain assessment 1 year postoperatively.

\section{Discussion}

The choice of the best surgical approach in the treatment of inguinal hernia is difficult. ${ }^{8}$ Laparoscopic repair provides very good results as it is associated with minimal postoperative pain, few wound infection rates, and quick return to daily activity. ${ }^{9}$ Even though method of mesh fixation might have an impact on recurrence rates, surgical site infections, postoperative chronic pain or quality-of-life, no accepted gold standard exists on whether, when and how to fix the mesh. ${ }^{10}$

This study was conducted to compare the outcome of using absorbable versus non-absorbable tacks for mesh fixation during TAPP as regard pain and recurrence. In our study there was no significant difference between the 2 groups as regard pain and recurrence.

In 2008, Taylor et al showed that tacking increases postoperative pain when compared to no fixation. ${ }^{11}$ Because of the Taylor et al study, the number of tacks used has dramatically decreased nationwide, with most people using only one or two tacks as adequate fixation. ${ }^{4,11}$ In 2011 , a study revealed that acute pain rate increased when more than 10 tacks were placed. ${ }^{12}$

In the present study, we used 3-5 tacks for mesh fixation for each hernia side repair and another 3-5 tacks for peritoneal closure in both groups so the number of tacks on each hernia side repair didn't exceed 10 tacks. The mean number of tacks was $16.87 \pm 3.22$ in group A and 19 (13-20) with mean $17.4 \pm 2.92$ in group B. This is comparable with other studies. In a study done by Agresta et al on 50 patients with bilateral inguinal hernia Agresta et al used 3 absorbable tacks for mesh fixation and 4-5 tacks for peritoneal closure on each side. ${ }^{13}$ In Sharma et al study, 4-8 tacks were used for each side for fixing meshes to Cooper's ligament and anterior abdominal wall and another 7- 9 more tacks had to be used for each side in the TAPP Group for repositioning both the flaps. Tacks used in the TAPP group were $16.53 \pm 1.28$ but the type of tacks was not mentioned in the study. ${ }^{14}$

In this study, we have found no recurrence in 30 patients during our follow-up period for 12 months. This is comparable to the results of Agresta et al study. ${ }^{13}$

The postoperative pain assessment results in this study are comparable to other studies. In a multicenter study assessing the use of absorbable tacks as regard postoperative pain revealed that at 1 month, $90 \%$ of patients were totally pain-free (VAS score: 0 ) and only ten patients reported low pain (VAS scores: 0.3-3.1). At 1 year, the pain described by those ten patients finally disappeared, $98 \%$ of patients were totally pain-free. ${ }^{15}$ In Agresta et al study, VAS score was $6 \pm 3$ after 12 month. ${ }^{13}$ In Sharma et al study, early post operative pain was assessed by VAS score which was $3.40 \pm 1.37$ and $0.93 \pm 0.64$ at $24 \mathrm{~h}$ and $48 \mathrm{~h}$ postoperative, respectively. ${ }^{14}$

Other results from this study were comparable to other studies on TAPP using tacks as regard operative time, intraoperative complications, hospital stay, postoperative complications and return to normal activity.

By comparing the mean operative time in both groups, we found that the mean operation time was $77.67 \pm 34.33$ minutes in group A and $80.2 \pm 23.88$ minutes in group $B$ and that's comparable to the mean operation time of the tackers group in a study comparing tackers to glue for mesh fixation where the mean operation time was

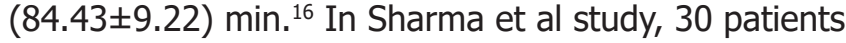


with bilateral hernia underwent TAPP with mean operating time $108.16 \pm 16.10 \mathrm{~min}$ (Range $=65-135$ $\min ) .{ }^{14}$

A single patient had intraoperative urinary bladder injury during peritoneal dissection in group $A$. The bladder was repaired laparoscopically with intracorporeal suturing and postoperative catherization for 2 weeks. This happened due to excessive dissection. Bladder injury is possible during peritoneal access but is rare, usually as the result of failure to decompress a distended bladder. Less commonly, injury is associated with a congenital bladder abnormality. Excessive dissection also may cause bladder injury. A minor injury can be treated conservatively with postoperative decompression via an indwelling catheter. When the injury is caused by a trocar, formal bladder repair is usually necessary. ${ }^{17}$

The mean postoperative hospital stay was $1.27 \pm 0.46$ days in group $A$ and $1.2 \pm 0.41$ days in group $B$. This is comparable to other studies; Agresta et al study where all procedures were performed as day cases and all the patients were discharged before $8 \mathrm{pm}$ in surgery day. ${ }^{13}$ In Sharma et al study, the mean hospital stay was $52.0 \pm 14.21 \mathrm{~h}^{14}$

Postoperative complications included inguinoscrotal edema; 2 patients in group $A$ and 1 patient in group $B$, all were treated conservatively within 2 weeks. One patient in group $B$ had port site infection and this was the only case of readmission for drainage. The patient was readmitted to the hospital 3 weeks after the operation with an infected port site. Drainage of the collection was done as a day case under general anaesthesia with no extension to the mesh site found and the postoperative period passed uneventful. Wound infection is a rare complication of groin herniorrhaphy (less than $5 \%)^{18}$

IIn this study, the mean time for return to normal activity was $5.33 \pm 1.68$ in group $A$ and $5.4 \pm 1.18$ in group B. In an Italian study on 100 patients who had a TAPP repair, their mean time to full activity return was 7.4 days. ${ }^{19}$ Sharma et al recorded mean time for return to work was $11.8 \pm 2.35$ days. ${ }^{14}$

Although there were no studies carried to compare the outcome of absorbable and non absorbable tacks in TAPP, studies comparing absorbable and non absorbable tacks in laparoscopic ventral and incisional hernia repair showed comparable results in terms of operative time, immediate postoperative pain, chronic pain and recurrence. ${ }^{20,21}$

This study is not without limitations. Mainly, the number of patients is low. The average follow-up of 12 months is reasonable for detecting early recurrences but not late recurrences. Other parameters as quality of life and cost were not included in the study.

\section{Conclusion}

Both non absorbable and absorbable tacks are feasible mesh fixation devices in laparoscopic transabdominal inguinal hernia repair and both have comparable results regarding postoperative pain and recurrence. Yet, longer follow up and further clinical trials are needed to determine the optimal method for mesh fixation.

\section{References}

1. Fridman A: Mesh fixation devices and techniques: A Review of the Literature. Bariatric Times. 2012; $9(6): 10-12$

2. Mayer $F$, Niebuhr $H$, Lechner $M$, et al: When is mesh fixation in TAPP-repair of primary inguinal hernia repair necessary? The register-based analysis of 11230 cases. Surgical Endoscopy. 2016; 30(10): 4363-4371.

3. Ge L, Tian J, Li L, et al: Mesh fixation methods in open inguinal hernia repair: A protocol for network meta-analysis and trial sequential analysis of randomized controlled trials. BMJ Open. 2015; 5(11): e009369.

4. Teng YJ, Pan SM, Liu YL, et al: A meta-analysis of randomized controlled trials of fixation versus nonfixation of mesh in laparoscopic total extraperitoneal inguinal hernia repair. 2011; 25(9): 2849-2858.

5. Brugger L, Bloesch M, Ipaktchi R, et al: Objective hypoesthesia and pain after transabdominal preperitoneal hernioplasty: A prospective, randomized study comparing tissue adhesive versus spiral tacks. Surg Endosc. 2012; 26(4):1079-1085.

6. Colak E, Ozlem N, Kucuk GO, Aktimur R, Kesmer $\mathrm{S}$, Yildirim K: Prospective randomized trial of mesh fixation with absorbable versus non absorbable tacker in laparoscopic ventral incisional hernia repair. Int J Clin Exp Med. 2015; 8(11): 2161121616

7. Silecchia G, Campanile FC, Sanchez L, Ceccarelli G, Antinori A, Ansaloni L, Olmi S, Ferrari GC, Cuccurullo D, Baccari P, Agresta F, Vettoretto N and Piccoli M: Laparoscopic ventral/ incisional hernia repair: Updated guidelines from the EAES and EHS endorsed Consensus Development Conference. Surg Endosc. 2015; 29: 2463-2484.

8. Pisanu A, Podda M, Saba A et al: Meta-analysis and review of prospective randomized trials comparing laparoscopic and Lichtenstein techniques in recurrent inguinal hernia repair. Hernia. 2015; 19: 355-358.

9. Karthikesalingam A, Markar S, Holt P, Praseedom R: Meta-analysis of randomized controlled trials comparing laparoscopic with open mesh repair of recurrent inguinal hernia. Br J Surg. 2010; 97: 4-11. 
10. Lederhuber $\mathrm{H}$, Stiede $\mathrm{F}$, Axer $\mathrm{S}$, et al: Mesh fixation in endoscopic inguinal hernia repair: Evaluation of methodology based on a systematic review of randomized clinical trials. Surg Endosc. 2017; 31: 437-442.

11. Taylor C, Layani L, Liew V, et al: Laparoscopic inguinal hernia repair without mesh fixation, early results of a large randomized clinical trial. Surg Endosc. 2008; 22(3): 757-762.

12. Belyansky I, Tsirline V, Klima D, Walters A, Lincourt A, Heniford T: Prospective, comparative study of postoperative quality of life in TEP, TAPP, and modified Lichtenstein repairs. Ann Surg. 2011; 254(5): 709-714.

13. Agresta F, Marzetti A, Verza LA, Prando D, Azabdaftari A, Rubinato L, Vacca U, Roveran A, Porfidia R, Vigna SAM: Laparoscopic TAPP inguinal hernia repair: Mesh fixation with absorbable tacks, initial experience. J Minim Invasive Surg Sci. 2016 May; 5(2): e35609.

14. Sharma D, Yadav K, Hazrah P, Borgharia S, Lal R, Thomas S: Prospective randomized trial comparing laparoscopic transabdominal preperitoneal (TAPP) and laparoscopic totally extra peritoneal (TEP) approach for bilateral inguinal hernias. International Journal of Surgery. 2015; 22: 110-117.

15. Lepere M, Benchetrit S, Bertrand JC, Chalbet JY, Combier JP, Detruit B, et al: Laparoscopic resorbable mesh fixation. Assessment of an innovative disposable instrument delivering resorbable fixation device: I-ClipTM. Final results of a prospective multicentre clinical trial. Hernia. 2008; 12: 177-183.

16. Burza A, Avantifiori R, Curinga R, Santini E, Delle $\mathrm{SP}$, Stipa F: Comparison between two different mesh fixation methods in laparoscopic inguinal hernia repair: Tacker vs. synthetic cyanoacrylate glue. Minerva Chirurgica. 2014; 69(6): 321-329.

17. Fitzgibbons R, Giobbie A, Gibbs J et al: Watchful waiting vs repair of inguinal hernia in minimally symptomatic men: A randomized clinical trial. JAMA. 2006; 295: 285-292.

18. Sanchez M FJ, Lozano GJ, Seco G JL: Antibiotic prophylaxis for hernia repair. Cochrane Database Syst Rev. 2012; 2: CD003769.

19. Sun $P, H u S B$, Cheng $X$ et al: Inguinal Hernia: Mesh Fixation. Hernia. 2015; 19(1): 157-159.

20. Bansal VK, Asuri K, Panaiyadiyan S, Kumar S, Subramaniam R, Ramachandran R, Sagar R, Misra MC: Comparison of Absorbable Versus Nonabsorbable Tackers in Terms of Long-term Outcomes, Chronic Pain, and Quality of Life After Laparoscopic Incisional Hernia Repair: A Randomized Study. Surg Laparosc Endosc Percutan Tech. 2016; 26(6): 476-483.

21. Khan RMA, Bughio M, Ali B, Hajibandeh $S$, Hajibandeh S: Absorbable versus non-absorbable tacks for mesh fixation in laparoscopic ventral hernia repair: A systematic review and metaanalysis. Int J Surg. 2018; 53: 184-192. 